and notifying medical providers of their study participation. In rare cases we have paid to transport patients from skilled nursing facilities to the clinic, which has reduced dropouts. In addition to the stroke participants, we have enrolled 7 healthy control participants using the EHR screening algorithm. DISCUSSION/SIGNIFICANCE OF IMPACT: Performing a longitudinal study in the early recovery phase following stroke is logistically challenging, but feasible. Difficulty in identifying participants with isolated motor impairment requires added effort to eliminate dropouts. Screening the EHR is an effective method to identify matched controls. Future metabolomics analysis of stored blood samples holds promise to identify biomarkers of stroke recovery and neural repair.

3159

Bone Turnover Biomarkers May Discriminate Low Bone Mineral Density in HIV-Infected Adults

Lauren Frances Collins ${ }^{1}$, Anandi Sheth ${ }^{1}$, Caitlin Moran ${ }^{1}$, Laura Ward ${ }^{1}$, Kehmia Titanji ${ }^{1}$, Kirk Easley ${ }^{1}$, Jeffrey Lennox ${ }^{1}$, M. Neale Weitzmann ${ }^{1}$ and Igho Ofotokun ${ }^{1}$

${ }^{1}$ Emory University

OBJECTIVES/SPECIFIC AIMS: Persons living with HIV (PLWH) are at increased risk for fragility bone disease. Current osteoporosis screening guidelines do not account for HIV status, and clinical risk assessment tools are not sensitive in PLWH. We examined the value of traditional osteoporosis risk factors, HIV-specific indices, and bone turnover biomarkers in predicting low bone mineral density (BMD) in PLWH. METHODS/STUDY POPULATION: Demographic and clinical characteristics, dual energy $\mathrm{X}$-ray absorptiometry (DXA)-derived BMD, HIV indices (viral load, CD4 count, antiretroviral therapy [ART]), and biomarkers of bone turnover (C-terminal telopeptide of collagen [CTx], osteocalcin [OCN]) were evaluated in a cross-sectional analysis of PLWH $(n=248)$ and HIV-controls $(n=183)$. The primary outcome was low BMD, defined as osteopenia or osteoporosis by WHO criteria. Multivariable logistic and modified Poisson regression models were used to assess associations between low BMD and covariates of interest. RESULTS/ ANTICIPATED RESULTS: Overall, median age was 44 years, $48 \%$ were male, $88 \%$ were black, median body mass index (BMI) was $28 \mathrm{~kg} / \mathrm{m} 2,72 \%$ smoked cigarettes, and 53\% used alcohol; characteristics did not differ by HIV status. PLWH had a mean CD4 of 408 cells/mm3, 55\% were ART-naïve, and $45 \%$ had viral suppression on ART. Overall, 25\% (109/431) had low BMD, including 31\% of PLWH compared to $16 \%$ of HIV-controls. In multivariable models, HIV was significantly associated with low BMD (aOR 2.46, 95\%CI 1.39-4.34; aRR 1.90, 95\%CI 1.18-3.07). Adjusting for HIV, three traditional risks- age, race, and BMI- were independently associated with low BMD in the full cohort. However, bone turnover markers, CTx and OCN, were better able to discriminate low vs. normal BMD in PLWH compared to HIV- controls. In PLWH, mean serum CTx was $23 \%$ higher in low vs. normal BMD (mean CTx difference= $0.06 \mathrm{ug} / \mathrm{mL}$ ); in HIV- controls, no association with BMD was observed (mean CTx difference $=0 \mathrm{ug} / \mathrm{mL}$ ). In PLWH, mean serum OCN was $38 \%$ higher in those with low vs. normal BMD (mean OCN difference $=2.48 \mathrm{ug} / \mathrm{mL}$ ); in HIV - controls, mean serum OCN was only $16 \%$ higher in those with low vs. normal BMD (mean OCN difference $=1.08 \mathrm{ug} / \mathrm{mL}$ ). DISCUSSION/SIGNIFICANCE OF IMPACT: In PLWH as opposed to HIV-controls, serum biomarkers reflecting a high bone turnover state, may discriminate individuals with low versus normal BMD. Because changes in biomarkers precede changes in $\mathrm{BMD}$, these markers should be explored further either alone or in combination with traditional risk assessment tools to improve early screening for osteoporosis in PLWH.

3517

\section{Cancer-Related Pain is a Predictor of In-hospital Opioid Overdose among Postoperative patients}

Nnaemeka E Onyeakusi ${ }^{1}$, Fahad Mukhtar ${ }^{2}$,

Adebamike Oshunbade ${ }^{3}$, Semiu Gbadamosi ${ }^{4}$, Adeyinka Adejumo ${ }^{5}$ and Jude C. Owoh 6

${ }^{1}$ BronxCare Hospital Center; ${ }^{2}$ St. Elizabeth's Hospital; ${ }^{3}$ University of Mississippi Medical Center; ${ }^{4}$ Florida International University; ${ }^{5}$ North Shore Medical Center and ${ }^{6}$ Quinnipiac University

OBJECTIVES/SPECIFIC AIMS: Our study's primary aim is to determine if there is an association between cancer-related pain among patients who underwent major elective procedures and postoperative opioid overdose. In addition, the relationship between cancerrelated pain in this population and inpatient mortality, total hospital charge and length of stay was assessed. METHODS/STUDY POPULATION: Our study sample consisted of adults 18 years and older who had at least one of eight elective procedures. Data was obtained from the National Inpatient Sample (NIS). Variables were identified using ICD-9 codes. Our primary predictor was cancerrelated pain while our primary outcome was opioid overdose. Secondary outcomes were inpatient mortality, length of stay and total charge. Propensity-matched regression models were employed in assessing the association between cancer-related pain and outcomes of interest. RESULTS/ANTICIPATED RESULTS: Among $4,085,355$ selected patients, $0.8 \%(n=2,665)$ had cancer-related pain while $99.92 \%(n=4,082,690)$ had no diagnosis of cancer-related pain. All subjects with cancer-related pain $(n=2,665)$ were successfully matched to subjects with no diagnosis of cancer-related pain in a 1:5 ratio yielding 13,325 controls. Patients with cancer-related pain had significantly higher odds of opioid overdose (aOR 4.82 [95\% CI [2.68-8.67]; p-value <0.0001) and inpatient mortality (aOR 1.39[1.11-1.74]; p-value 0.0043). Patients with cancer-related pain were also likely to stay significantly longer in the hospital (12.76 days vs. 7.88 days) with significantly higher total hospital charges $(\$ 140,220$ vs. $\$ 88,316)$. DISCUSSION/SIGNIFICANCE OF IMPACT: Pain is a common complication of cancer pathogenesis, diagnosis or treatment. Though a rare outcome, opioid overdose could lead to undesirable outcomes. Cancer patients undergo invasive diagnostic and therapeutic procedures as part of their cancer management or for conditions not related to their primary cancer diagnosis. Safety measures including alternatives to opioids are recommended to prevent the poor clinical outcomes and higher healthcare utilization indices associated with opioid overdose in this population.

3445

Cannabis use and risk of $\mathrm{H}$. pylori infection; analysis of inpatients and residents of the US.

Adeyinka Charles Adejumo ${ }^{1}$ and Terence Ndonyi Bukong

${ }^{1}$ North Shore Medical Center

OBJECTIVES/SPECIFIC AIMS: Cannabinoids suppress gastric acid secretion, ameliorate gastric inflammation, and promote gastric ulcer healing, all of which are triggered by $\mathrm{H}$ pylori ( $\mathrm{Hp}$ ). Our aim was to determine the relationship between cannabis use and: 1) $\mathrm{H}$ pylori infection (HPI) among community residents 2) clinical 